\title{
Was Albert Einstein Wrong on Quantum Physics?
}

\author{
Mani L. Bhaumik \\ Department of Physics and Astronomy, University of California, Los Angeles, California, USA \\ E-mail:bhaumik@physics.ucla.edu \\ Editors: Danko Georgiev \& Chris C. King \\ Article history: Submitted on November 24, 2015; Accepted on December 7, 2015; Published on December 11, 2015.
}

\begin{abstract}
A
lbert Einstein is considered by many physicists as the father of quantum physics in some sense. Yet there is an unshakable view that he was wrong on quantum physics. Although it may be a subject of considerable debate, the core of his allegedly wrong demurral was the insistence on finding an objective reality underlying the manifestly bizarre behavior of quantum objects. The uncanny waveparticle duality of a quantum particle is a prime example. In view of the latest developments, particularly in quantum field theory, the objections of Einstein are substantially corroborated. Careful investigation suggests that a travelling quantum particle is a holistic wave packet consisting of an assemblage of irregular disturbances in quantum fields. It acts as a particle because only the totality of all the disturbances in the wave packet yields the energymomentum with the mass of a particle, along with its other conserved quantities such as charge and spin. Thus the wave function representing a particle is not just a fictitious mathematical construct but embodies a reality of nature as asserted by Einstein.

Quanta 2015; 4: 35-42.
\end{abstract}

(c) $(9)$ This is an open access article distributed under the terms of the Creative Commons Attribution License CC-BY-3.0, which permits unrestricted use, distribution, and reproduction in any medium, provided the original author and source are credited.

\section{Introduction}

This year we celebrate with much aplomb the centenary of Einstein's unveiling of his ingenious General Theory of Relativity, although its seed was sown in 1905. In the same Annus Mirabilis, he also seeded the other seminal breakthrough of the 20th century: quantum mechanics. He is granted undisputed credit for the theory of relativity, but receives only guarded recognition for his essential contribution to the quantum revolution. In fact, there is a general impression that Einstein lost the debate on quantum physics. As we honor him for relativity, it is fitting to ask whether the legendary star of relativity was indeed wrong on quantum physics.

Einstein was the first physicist to support the veracity of Max Planck's radical postulate of quanta of energy [1,2]. Although the energy quanta were proposed in 1900, after years of frustration in formulating his law of black body radiation, Planck himself did not seem to believe in their actual existence. Even more than a decade later in 1913, in a petition recommending Einstein to be a member of the Prussian Academy of Sciences, Planck (together with Walther Nernst, Heinrich Rubens, and Emil Warburg) made a patronizing remark

That [Einstein] may sometimes have missed the target in his speculations, as, for example, in his hypothesis of light quanta, cannot really be held too much against him, for it is not possible to introduce fundamentally new ideas, even in the most exact sciences, without occasionally taking a risk. [3, p.44] 
Nernst referred to the light quanta as "probably the strangest thing ever thought up." But Einstein daringly peered through the veil.

Essentially, as early as in 1909 in his Salzburg address [4. p.321], Einstein had predicted that physics would have to reconcile itself to a duality in which light could be regarded as both wave and particle. And at the first Solvay Conference in 1911, he had declared that

these discontinuities, which we find so distasteful in Planck's theory, seem really to exist in nature. [4, p.608]

So, it was in fact Einstein who fostered the innovative notion of the wave-particle duality by asserting the real existence of quanta of radiation or photons, which eventually would open the door for him to his sole Nobel Prize for the photoelectric effect. Following his elicitation, young Louis de Broglie in his $\mathrm{PhD}$ thesis extended the concept to matter particles with crucial and enthusiastic support from Einstein.

To de Broglie's thesis advisor Langevin, the idea of a matter wave seemed far-fetched. So, he sent a skeptical note to his friend Einstein requesting that, "although the thesis is a bit strange, could he see if it was still worth something." Einstein replied with a glowing recommendation

Louis de Broglie's work has greatly impressed me. He has lifted a corner of the great veil. In my work I [have recently] obtained results that seem to confirm his. [5, p.242]

Later Einstein admitted to Isidor Isaac Rabi that he indeed thought about the equation for matter waves before de Broglie but did not publish it since there was no experimental evidence for it [5, p.252]. De Broglie expressed his appreciation by writing

As M. Langevin had great regard for Einstein, he counted this opinion greatly, and this changed a bit his opinion with regard to my thesis. [5, p.250]

Shortly after reading de Broglie's dissertation, Einstein began suggesting to physicists to look in earnest for an evidence of the matter wave. Soon, Clinton Davisson and Lester Germer furnished proof with the accidental discovery of electron waves in observing a diffraction pattern in a nickel crystal [6].

In the meantime Erwin Schrödinger, "inspired by L. de Broglie ... and by brief, yet infinitely far-seeking remarks of A. Einstein" [7, p.211], formulated the wave mechanics of quantum physics, which turned out to be equivalent to the rather abstract matrix mechanics devised by Werner
Heisenberg at about the same time. Is it then any wonder that eminent physicists like Leonard Susskind [8, p.xi] consider Einstein to be the father of quantum physics in some sense?

Yet, volumes have been written on Einstein's objection to the implications of quantum physics, particularly to the elements of uncertainty, probability, and nonlocality associated with it. There is no question that, as a true scientist, Einstein accepted the extraordinary success and the spectacular results of quantum physics. Can we discern, then, from the very extensive debates and discussions, what was the primary concern of Einstein in his objection to the interpretation of quantum physics? While there can be endless deliberations on this point, why not accept Einstein's own pronouncement on the subject? "At the heart of the problem," Einstein said of quantum mechanics, "is not so much the question of causality but the question of realism" [4, p.460].

Niels Bohr was content with his postulate of complementarity of wave-particle duality, emphasizing there is no single underlying reality that is independent of our observation. "It is wrong to think that the task of physics is to find out how nature is," Bohr declared. "Physics concerns what we can say about nature" [4, p.333]. Einstein derided this pronouncement as an almost religious delirium. He firmly believed there was an objective reality that existed whether or not we could observe it [4, p.334].

Most contemporary physicists part company with Einstein invoking that it would be futile to look for reality, which becomes totally obscure under the thick smoke of the heavy artillery of Hilbert space necessary to deal with particles in quantum mechanics. It is a daunting task indeed to discern any reality in the thickets of a configuration space! However, if each single particle comprising the ensemble of Hilbert spaces (Fock space) can be shown to have an objective reality individually, would not it be reasonable to infer that the ensemble in Fock space will also have realism even though one may not be able to decipher it?

Here, we present a credible allocution in favor of the existence of a physical reality behind the wave function at the core of quantum physics. This is primarily anchored on the incontrovertible physical evidence that all electrons in the universe are exactly alike. We provide reasonable support to show that the wave function of quantum mechanics is not just a conjured mathematical paradigm, but there is an objective reality underlying it, thus justifying Einstein's primary concern of the question of realism.

The answer to the longstanding puzzle of why all electrons are exactly identical in all respects, a feature eventually found to be shared by all the other fundamental particles as well, was finally provided by the quantum field theory of the Standard Model of particle physics 
constructed by combining Einstein's special theory of relativity with quantum physics, which evolved from his innovative contributions.

Quantum field theory has successfully explained almost all experimental observations in particle physics and correctly predicted a wide range of phenomena with impeccable precision. By way of many experiments over the years, the quantum field theory of the Standard Model has become recognized as a well-established theory of physics. Although one might argue that the Standard Model accurately describes the phenomena within its domain, it is still incomplete since it does not include gravity, dark matter, dark energy, neutrino oscillations and others. However, because of its astonishing success so far, whatever deeper physics may be necessary for its completion would very likely extend its scope without retracting the current fundamental depiction.

\section{Nature of Primary Reality Portrayed by Quantum Field Theory}

In order to fully grasp the deeper nature of the relativistic quantum fields, one has to go through the rather esoteric mathematical formalism of the quantum field theory. Nevertheless, its essence can be understood in terms of a narrative. Quantum field theory has uncovered a fundamental nature of reality, which is radically different from our daily perception. Our customary ambient world is very palpable and physical. But quantum field theory asserts this is not the primary reality. The fundamental particles involved at the underpinning of our daily physical reality are only secondary. Each fundamental particle, whether it is a boson or a fermion, has its corresponding underlying quantum field from which it originates [9-12]. The particles are excitations of their respective underlying quantum fields possessing propagating states of discrete energies, and it is these which constitute the primary reality. For example, a photon is a quantum of excitation of the photon field (aka electromagnetic field), the electron is a quantum of the electron quantum field, a quark is a quantum of the quark quantum field, and so on for all the fundamental particles of the universe. Inherent quantum fluctuations are also a distinct characteristic of a quantum field. Thus, quantum field theory substantiates the profoundly counter intuitive departure from our normal perception of reality to reveal that the foundation of our tangible physical world is something totally abstract, comprising of continuous quantum fields that create discrete local excitations we call particles.

By far, the most phenomenal step forward made by quantum field theory is the stunning prediction that the primary ingredient of everything in this universe is present in each element of spacetime ( $x, y, z, t)$ of this immensely vast universe [13, p.74]. These ingredients are the underlying quantum fields. We also realize that the quantum fields are alive with quantum activity. These activities have the unique property of being completely spontaneous and utterly unpredictable as to exactly when a particular event will occur [13, p.74]. Furthermore, some of the quantum fluctuations occur at mind-boggling speeds with a typical time period of $10^{-21}$ seconds or less. In spite of these infinitely dynamic, wild fluctuations, the quantum fields have remained immutable, as evinced by their Lorentz invariance, essentially since the beginning and throughout the entire visible universe encompassing regions, which are too far apart to have any communication even with the speed of light. This is persuasively substantiated by the experimental observation that a fundamental particle such as an electron has exactly the same physical properties, be its rest mass, charge or spin, irrespective of when or where the electron has been created, whether in the early universe, through astrophysical processes over the eons or in a laboratory today anywhere in the world. Such a precise match between theory and observation infuses immense confidence on our approach.

\section{Quantum Particle in Motion}

As elucidated above, an electron represents a propagating discrete quantum of the underlying continuous electron field. In other words, an electron is a quantized wave (or a ripple) of the electron quantum field, which acts as a particle because of its well-defined energy, momentum, and mass, which are conserved fundamentals of the electron. However, even a single electron, in its reference frame, is never alone. It is unavoidably subjected to the perpetual fluctuations of the quantum fields.

When an electron is created instantaneously from the electron quantum field, its position would be indefinite since a regular ripple with a very well-defined energy and momentum is represented by a delocalized periodic function. But the moment the electron comes into existence, it starts to interact with all the other quantum fields facilitated by quantum fluctuations of the fields. For example, the presence of the electron creates a disturbance in the photon (electromagnetic) quantum field. Assisted by a fleeting quantum fluctuation, the disturbance in the photon field can momentarily appear as what is commonly known as a spontaneously emitted virtual photon.

To conserve momentum, the electron would recoil with momentum equal and opposite to that of the photon. A quantum fluctuation of energy $\Delta E$ will provide the kinetic energy for the recoil of the electron as well as the 
A
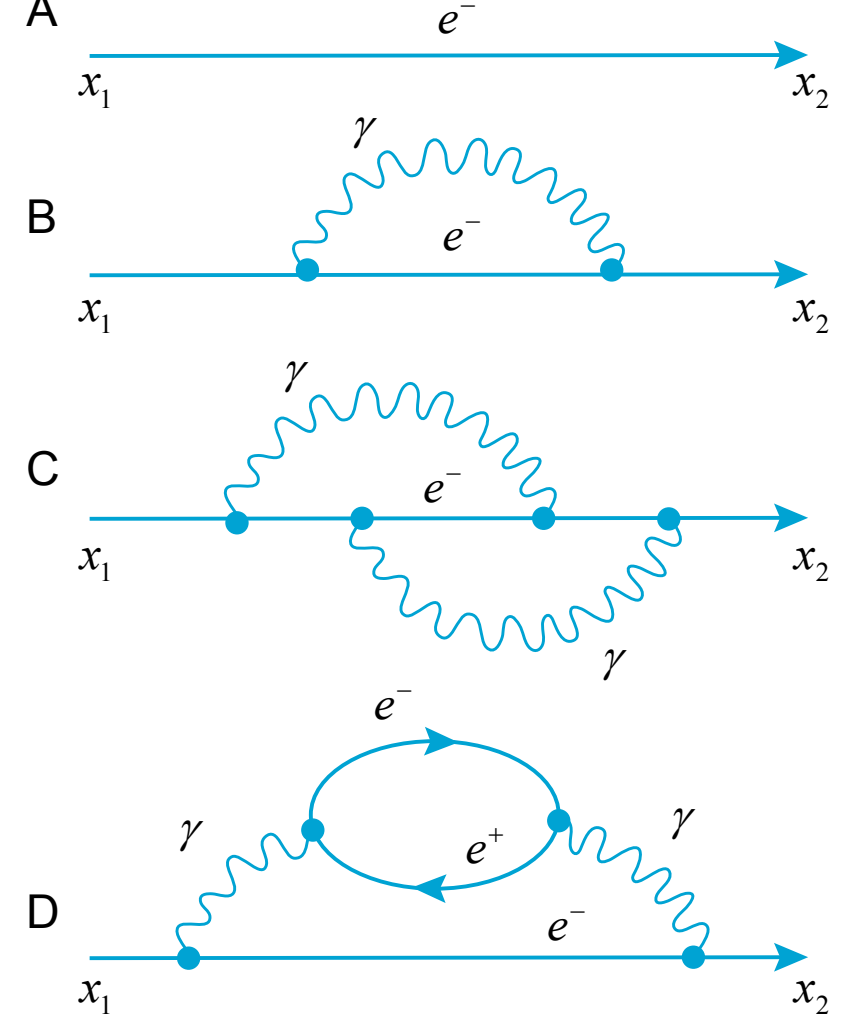

Figure 1: Feynman diagrams showing some of the various interactions between quantum fields during transit of a quantum particle like an electron $e^{-}$from $x_{1}$ to $x_{2}(A)$. (B) Interaction of an electron with the photon field, which is commonly described as the emission of a virtual photon $\gamma$ by the electron and then reabsorbing it. (C) Emission of two photons and re-absorption by the electron. The photon in turn can create disturbances in the various quantum fields involving a charge. The virtual photon can emit an electron-positron pair $e^{-}+e^{+}$as shown in (D), a muon-anti muon pair, a quark-antiquark pair, etc.

energy of the photon for a time $\Delta T \sim \hbar / \Delta E$. During this transitory moment, the electron by creating a disturbance in the photon field becomes a disturbed ripple itself and therefore ceases to be a normal particle on its own.

All these disturbances are elegantly depicted by Feynman diagrams (see Fig. 1), which also aid in calculating the interaction energies among the various quantum fields. The disturbance in the photon (electromagnetic) field in turn can cause disturbances in all the electrically charged quantum fields, like the electron, muon and the various quark fields. Generally speaking, in this manner, every quantum particle spends some time as a mixture of other virtual particles in all possible ways.

The quantum fluctuations continually and prodigiously create virtual electron-positron pairs in a volume surrounding the electron. "Each pair passes away soon after it comes into being, but new pairs are consistently boiling up to establish an equilibrium distribution" [14, p.404]. Although each pair has a fleeting existence, on average there is a very significant amount of these pairs to impart a remarkably sizable screening of the bare charge of the electron.

Likewise, though any individual disturbances in the fields or the virtual particles due to quantum fluctuations have an ephemeral existence, there ought to be an equilibrium distribution of such disturbances present at any particular time affecting other aspects of the electron. The effect of these disturbances is very well established in phenomena such as the Lamb shift and the anomalous $g$-factor of the electron spin.

The electron spin $g$-factor has been measured to a precision of better than one part in a trillion, compared to the theoretically calculated value that includes Feynman diagrams up to four loops [15]. Therefore it would be reasonable to assume that the equilibrium distribution of disturbances present at any particular time due to all quantum fields involved will be very stable in spite of their fleeting existence.

Let us recall that an electron is a quantized ripple of the electron quantum field, which acts as a particle because it travels holistically with its conserved quantities always sustained as a unit. However, due to interactions of the particle with all the other quantum fields, substantially equivalent to those involved in the Lamb shift and the observed spin $g$-factor, the ripple in fact becomes very highly distorted immediately after its creation since the quantum fluctuations prompting the interactions of the quantum fields have a typical time period of $10^{-21}$ seconds. Consequently, the electron ceases to be a ripple of single frequency and becomes a highly deformed localized travelling pulse.

It is well known that such a pulse, no matter how deformed, can be expressed by a Fourier integral with weighted linear combinations of simple periodic wave forms like trigonometric functions, briefly mentioned by the author in a previous work [16]. The result would be a wave packet or a wave function that represents a fundamental reality of the universe. Such a wave function would be smooth and continuously differentiable, especially using imaginary numbers in the weighted amplitude coefficients. The wave function $\psi(x)$ will be given by the Fourier integral

$$
\psi(x)=\frac{1}{\sqrt{2 \pi}} \int_{-\infty}^{+\infty} \tilde{\psi}(k) e^{\imath k x} d k
$$

where $\tilde{\psi}(k)$ is a function that determines the amount of each wave number component $k=2 \pi / \lambda$ that gets added to the combination.

From Fourier analysis, we also know that the spatial wave function $\psi(x)$ and the wave number function $\tilde{\psi}(k)$ are a Fourier transform pair. Therefore, we can find the 
wave number function through the Fourier transform of $\psi(x)$ as

$$
\tilde{\psi}(k)=\frac{1}{\sqrt{2 \pi}} \int_{-\infty}^{+\infty} \psi(x) e^{-l k x} d x
$$

Thus the Fourier transform relationship between $\psi(x)$ and $\tilde{\psi}(k)$, where $x$ and $k$ are known as conjugate variables, can help us determine the frequency or the wave number content of any spatial wave function.

\section{The Uncertainty Principle}

The Fourier transform correlations between conjugate variable pairs have powerful consequences since these variables obey the uncertainty relation

$$
\Delta x \Delta k \geq \frac{1}{2}
$$

where $\Delta x$ and $\Delta k$ relate to the standard deviations $\sigma_{x}$ and $\sigma_{k}$ of the wave packet. This is a completely general property of a wave packet with a reality of its own and is in fact inherent in the properties of all wave-like systems. It becomes important in quantum mechanics because of de Broglie's introduction of the wave nature of particles by the relationship $p=\hbar k$, where $p$ is the momentum of the particle. Substituting this in the general uncertainty relationship of a wave packet, the intrinsic uncertainty relation in quantum mechanics becomes

$$
\Delta x \Delta p \geq \frac{1}{2} \hbar
$$

This uncertainty relationship has been misunderstood with a rather analogous observer effect, which posits that measurement of certain systems cannot be made without affecting the system. In fact, Heisenberg offered such an observer effect in the quantum domain as a "physical explanation" of quantum uncertainty, a maxim that now popularly goes by the name Heisenberg's uncertainty principle. But the uncertainty principle actually states a fundamental property of quantum systems, and is not a statement about the observational indeterminacy as was emphasized by Heisenberg. In fact, some recent studies [17-19] highlight important fundamental difference between uncertainties in quantum systems and the limitation of measurement in quantum mechanics.

Einstein's fundamental objection to the Copenhagen interpretation was its assertion that any underlying reality of the uncertainties was irrelevant and should be acceptable under the veil of complementarity. We have established that there is indeed an intrinsic uncertainty induced by the wave behavior that is as much a fact of nature as the electron itself, and that it traces its origin back to the wave-particle duality first envisioned by Einstein as a reality.

\section{Role of Probability in}

\section{Measurement}

Having been an expert on statistical mechanics, Einstein was no stranger to probability. In fact, he was not opposed to the probabilistic implication of quantum physics. As Wolfgang Pauli reported to Max Born

Einstein does not consider the concept of 'determinism' to be as fundamental as it is frequently held to be (as he told me emphatically many times) ... In the same way, he disputes that he uses as criterion for the admissibility of a theory the question: 'Is it rigorously deterministic?' [20, p.221]

As always, he was essentially searching for realism behind the probabilistic outcome in quantum physics.

In Section 3, we have argued that quantum fields are the primary objective reality. A quantum, or more specifically a quantized ripple of the field, has the characteristics of a particle. But at the very instant of creation, the energy-momentum being fixed, the ripple has only a single frequency and is therefore totally delocalized, which is not a characteristic of a particle. Immediately after the instant of creation, though, the ripple starts to interact with all the other quantum fields. The distorted ripple is equivalent to a localized wave packet that starts to look like a particle, but only the totality of all the disturbances possesses the properties of the particle. The disturbances in the wave packet travel holistically as a unit and thereby acts as a particle. When the wave function is reduced by measurement or otherwise, again the totality of all the field disturbances must be taken as a unit because of the conserved quantities of the particle. The wave function disappears everywhere else, except where it is reduced.

It should now be evident that the random disturbances caused by the intrinsic quantum fluctuations of the underlying field are the reason that a quantum particle such as an electron is always associated with a wave function. Such a wave function is by no means simply a mathematical construct as currently assumed by many physicists. It represents the totality of all the interactions in the various quantum fields caused by the presence of the electron and facilitated by quantum fluctuations. In other words, a quantum particle like an electron in motion is a travelling holistic wave packet consisting of the irregular disturbances of the various quantum fields. It is holistic in the sense that only the combination of the disturbances in the electron field together with those in all the other fields always maintains a well-defined energy and momentum with an electron mass, since they are conserved quantities for the electron as a particle. 
Since a particle like an electron in motion is represented by a wave function, its kinematics cannot be described by the classical equations of motion. Instead, it requires the use of an equation like the Schrödinger equation for a non-relativistic particle

$$
i \hbar \frac{\partial}{\partial t} \psi(x, t)=-\frac{\hbar}{2 m} \nabla^{2} \psi(x, t)+V(x) \psi(x, t)
$$

where $V(x)$ is the classical potential and the wave function $\psi(x, t)$ is normalized

$$
\int_{-\infty}^{+\infty} \psi^{*}(x, t) \psi(x, t) d x=1
$$

The wave function evolves impeccably in a unitary way. However, when the particle inevitably interacts with a classical device such as a measuring apparatus, the wave function undergoes a sudden discontinuous change known as the wave function collapse. Although it is an essential postulate of the Copenhagen interpretation of quantum mechanics, the phenomenon has long been perplexing to the physicists [21, p.786]. However, a behavior like this would be a natural consequence of the distinctive nature of a quantum particle described in Section 3. In support of this notion, the holistic nature of the wave function is presented as evidence. In a measurement, this holistic nature becomes obvious since the appearance of the particle in one place prevents its appearance in any other place.

Contrary to the waves of classical physics, the wave function cannot be subdivided during a measurement. This is specifically because the combination of all the disturbances comprising the wave function possesses a well-defined energy and momentum with the mass of the particle. Consequently, only the totality of the wave function must be taken for detection, causing its disappearance everywhere except where the particle is measured. This inescapable fact could hint at a solution to the well-known measurement paradox.

It has been indeed very difficult to understand why, after a unitary evolution, the wave function suddenly collapses upon measurement or a similar other reductive interaction. The holistic nature of the wave function described above seems to offer a plausible explanation. Parts of the wave function that might spread to a considerably large distance can also terminate instantaneously by the process involved in a plausible quantum mechanical Einstein-Rosen bridge [16,22] and experimentally demonstrated in quantum entanglement of a single photon [23].

Thus, the very weave of our universe appears to support the objective reality of the wave function, which represents a natural phenomenon and not just a mathematical construct. We also observe that while the wave nature predominates as a very highly disturbed ripple of the quantum field before a measurement, the particle aspect becomes paramount upon measurement.

Because of the wave nature of the particle, the position where the wave packet would land is guided by the probability density $|\psi|^{2}$ given by the Born rule. It is only fitting to note that Born followed Einstein in this regard as he stated in his Nobel lecture

Again an idea of Einstein's gave me the lead. He had tried to make the duality of particleslight quanta or photons-and waves comprehensible by interpreting the square of the optical wave amplitudes as probability density for the occurrence of photons. This concept could at once be carried over to the $\psi$-function: $|\psi|^{2}$ ought to represent the probability density for electrons (or other particles). [24, p.262]

The exact mechanism by which the wave function collapses is still hotly debated. The most popular version envisions the wave function becoming entangled with the constituents of the detector and decohering very quickly due to the irreversible thermal motion. One of the principal contributors to the theory of decohernce, Wojciech Zurek contends [25] that the Born rule can actually be derived from the theory of decoherence as opposed to being a mere postulate of quantum theory. There is indeed some support for his contention [26].

The Copenhagen interpretation also requires a conscious observer as an essential part of its formalism, which posits that the reality of a quantum system does not exist until an observer takes part in its detection, thereby causing the wave function to collapse. Einstein objected to this view with his famous query, does "the moon exist only when I look at it?" Although an observer can indeed bring out a particular reality, the fact that the universe, which is quantum at the core, developed to a mature state eons before any manner of conscious observer could appear supports Einstein's skepticism. His contention was that an objective reality should always be present irrespective of measurement.

In contrast, the supporters of the Copenhagen interpretation did not feel it was necessary to delve any further than acceptance of the wave-particle duality and its consequent uncertainty as a principle of complementarity. In view of the nature of reality discussed in this paper, there is no genuine conflict between Einstein's insistence of an underlying reality and the doctrine of complementarity in the Copenhagen interpretation. The intense debate in the pioneering period of quantum physics would appear to be superfluous in view of the nature of the universe revealed to us today. Then the question of who won the debate would have been redundant. 
Much has been said about how Einstein got it wrong in the Einstein-Podolsky-Rosen paper [27], in which he attempted to show that quantum mechanics was incomplete and would need further elucidation in the future. For two entangled particles separated by a great distance, Einstein believed there could be no immediate effect to the second particle as a result of anything that was done to the first, since that would violate special relativity. Quantum mechanics predicted otherwise, which he called, "spooky action at a distance." Contrary to Einstein's expectation, all experimental results so far support nonlocality [28-31]. Experimental evidence consistently shows that when two particles undergo entanglement, whatever happens to one of the particles can instantly affect the other, even if the particles are separated by an arbitrarily large distance!

Has Einstein's dream of an objective reality been shattered by these experiments? Not necessarily. It is hard to imagine Einstein would have given up just yet. He would still believe that some deeper reality, perhaps something stranger, lay behind the "spooky action" and certainly that is a reasonable possibility.

Experts such as Maldacena and Susskind [32] postulate that $\mathrm{ER}=\mathrm{EPR}$, namely quantum entangled particles (in Einstein-Podolsky-Rosen state [27]) are connected by a wormhole (Einstein-Rosen bridge [22]), implying there is an as yet unknown quantum version of a classical wormhole that permits quantum mechanical nonlocality. There is also a possibility that the quantum fluctuations of the fields are themselves entangled facilitating a quantum wormhole [16]. So there still could be an element of objective reality behind quantum entanglement.

In any case, quantum entanglement violates neither causality nor special relativity, since no classical bit of information can be sent using it [33, 34]. Einstein could still have the ultimate chuckle, notwithstanding the fact that some unexpected, specific form of instantaneous action at a distance has been experimentally demonstrated. More so, because in a serendipitous way, the discovery of quantum entanglement has opened up some groundbreaking applications such as quantum cryptography, quantum computing, and quantum teleportation, which have become areas of very active research. As a consequence, the Einstein-Podolsky-Rosen paper [27] has turned out to be a cornerstone in our understanding of quantum physics. If this represents a misstep, it is a fortuitous one that has yielded and will continue to yield a great bounty.

\section{Acknowledgement}

The author wishes to thank Zvi Bern and James Ralston for important discussions.

\section{References}

[1] Planck M. Ueber das Gesetz der Energieverteilung im Normalspectrum. Annalen der Physik 1901; 309 (3): 553-563. doi : 10.1002/andp. 19013090310

[2] Einstein A. Über einen die Erzeugung und Verwandlung des Lichtes betreffenden heuristischen Gesichtspunkt. Annalen der Physik 1905; 17 (6): 132-148. doi : 10.1002/andp.19053220607

[3] Jammer M. The Conceptual Development of Quantum Mechanics. New York: McGraw-Hill, 1966.

[4] Isaacson W. Einstein: His Life and Universe. New York: Simon \& Schuster, 2008.

[5] Stone AD. Einstein and the Quantum: The Quest of the Valiant Swabian. Princeton, New Jersey: Princeton University Press, 2013.

[6] Davisson CJ, Germer LH. Reflection of electrons by a crystal of nickel. Proceedings of the National Academy of Sciences 1928; 14 (4): 317-322. doi: 10.1073/pnas.14.4.317

[7] Moore WJ. Schrödinger: Life and Thought. Cambridge: Cambridge University Press, 1989.

[8] Susskind L, Friedman A. Quantum Mechanics: The Theoretical Minimum. What You Need to Know to Start Doing Physics. New York: Basic Books, 2014.

[9] Susskind L. Particle Physics: 1. Basic Concepts. Stanford Continuing Studies 2009: https://www.youtube.com/view_play_ list? $\mathrm{p}=768 \mathrm{E} 1383 \mathrm{EA79C603}$

[10] Kuhlmann M. Quantum field theory. In: Stanford Encyclopedia of Philosophy, Zalta EN, Nodelman U, Allen C (editors), Stanford, California: Stanford University, 2012, http://plato.stanford, edu/entries/quantum-field-theory/

[11] Srednicki M. Quantum Field Theory. Cambridge: Cambridge University Press, 2006. http://web. physics.ucsb.edu/ mark/qft.html

[12] Zee A. Quantum Field Theory in a Nutshell. Princeton, New Jersey: Princeton University Press, 2003.

[13] Wilczek FA. The Lightness of Being: Mass, Ether, and the Unification of Forces. New York: Basic Books, 2008. 
[14] Wilczek FA. Fantastic Realities: 49 Mind Journeys And a Trip to Stockholm. Singapore: World Scientific, 2006. doi: 10.1142/6019

[15] Brodsky SJ, Franke VA, Hiller JR, McCartor G, Paston SA, Prokhvatilov EV. A nonperturbative calculation of the electron's magnetic moment. Nuclear Physics B 2004; 703 (1-2): 333-362. arXiv: hep-ph/0406325, doi:10.1016/j .nuclphysb. 2004.10 .027

[16] Bhaumik ML. Reality of the wave function and quantum entanglement. 2014: arXiv: 1402.4764

[17] Rozema LA, Darabi A, Mahler DH, Hayat A, Soudagar Y, Steinberg AM. Violation of Heisenberg's measurement-disturbance relationship by weak measurements. Physical Review Letters 2012; 109 (10): 100404. arXiv: 1208.0034, doi:10.1103/ PhysRevLett.109.100404

[18] Erhart J, Sponar S, Sulyok G, Badurek G, Ozawa M, Hasegawa Y. Experimental demonstration of a universally valid error-disturbance uncertainty relation in spin measurements. Nature Physics 2012; 8 (3): 185-189. arXiv:1201.1833, doi:10.1038/ nphys2194

[19] Ozawa M. Heisenberg's original derivation of the uncertainty principle and its universally valid reformulations. 2015: arXiv: 1507.02010

[20] Born M, Einstein A. The Born-Einstein Letters: Correspondence Between Albert Einstein and Max and Hedwig Born from 1916-1955, with Commentaries by Max Born. Born I (translator), London: Macmillan, 1971. http://archive.org/ details/TheBornEinsteinLetters

[21] Penrose R. The Road to Reality: A Complete Guide to the Laws of the Universe. London: Jonathan Cape, 2004.

[22] Einstein A, Rosen N. The particle problem in the general theory of relativity. Physical Review 1935; 48 (1): 73-77. doi : 10.1103/PhysRev . 48.73

[23] Fuwa M, Takeda S, Zwierz M, Wiseman HM, Furusawa A. Experimental proof of nonlocal wavefunction collapse for a single particle using homodyne measurements. Nature Communications 2015; 6: 6665. arXiv:1412.7790, doi:10. 1038/ncomms 7665
[24] Born M. The statistical interpretation of quantum mechanics. Nobel Lecture, December 11, 1954. http://wwW.nobelprize.org/ nobel_prizes/physics/laureates/1954/ born-lecture.pdf

[25] Zurek WH. Probabilities from entanglement, Born's rule $p_{k}=\left|\psi_{k}\right|^{2}$ from envariance. Physical Review A 2005; 71 (5): 052105. arXiv:quant-ph/ 0405161, doi: 10.1103/PhysRevA.71.052105

[26] Schlosshauer M, Fine A. On Zurek's derivation of the Born rule. Foundations of Physics 2005; 35 (2): 197-213. arXiv:quant-ph/0312058, doi: 10.1007/s10701-004-1941-6

[27] Einstein A, Podolsky B, Rosen N. Can quantummechanical description of physical reality be considered complete? Physical Review 1935; 47 (10): 777-780. doi:10.1103/PhysRev.47.777

[28] Aspect A, Dalibard J, Roger G. Experimental test of Bell's inequalities using time-varying analyzers. Physical Review Letters 1982; 49 (25): 1804-1807. doi:10.1103/PhysRevLett.49.1804

[29] Gröblacher S, Paterek T, Kaltenbaek R, Brukner Č, Żukowski M, Aspelmeyer M, Zeilinger A. An experimental test of non-local realism. Nature 2007; 446 (7138): 871-875. arXiv:0704.2529, doi:10. 1038/nature05677

[30] Peruzzo A, Shadbolt PJ, Brunner N, Popescu S, O'Brien JL. A quantum delayed choice experiment. Science 2012; 338 (6107): 634-637. arXiv: 1205. 4926, doi: $10.1126 /$ science. 1226719

[31] Shadbolt P, Mathews JCF, Laing A, O'Brien JL. Testing foundations of quantum mechanics with photons. Nature Physics 2014; 10 (4): 278-286. doi: 10.1038/nphys2931

[32] Maldacena J, Susskind L. Cool horizons for entangled black holes. Fortschritte der Physik 2013; 61 (9): 781-811. arXiv:1306.0533, doi:10.1002/ prop.201300020

[33] Vedral V. Quantum entanglement. Nature Physics 2014; 10 (4): 256-258. doi : 10.1038/nphys2904

[34] Brukner Č. Quantum causality. Nature Physics 2014; 10 (4): 259-263. doi : 10.1038/nphys2930 